\title{
Epidemiological evidence of higher susceptibility to vCJD in the
} young

\author{
Pierre-Yves Boëlle*1, Jean-Yves Cesbron² and Alain-Jacques Valleron ${ }^{1}$
}

Address: ${ }^{1}$ INSERM U444, Assistance Publique Hôpitaux de Paris, Université Pierre et Marie Curie, Paris, France and 2Immunité Anti-Infectieuse JE 2236, UFR de Médecine de Grenoble, Université Joseph Fourier, Grenoble, France

Email: Pierre-Yves Boëlle* - boelle@u444.jussieu.fr; Jean-Yves Cesbron - jean-yves.cesbron@ujf-grenoble.fr; AlainJacques Valleron - valleron@u444.jussieu.fr

* Corresponding author

Published: 10 August 2004

BMC Infectious Diseases 2004, 4:26 doi:10.1 186/1471-2334-4-26
Received: 12 February 2004

Accepted: 10 August 2004

This article is available from: http://www.biomedcentral.com/I47/-2334/4/26

(C) 2004 Boëlle et al; licensee BioMed Central Ltd.

This is an open-access article distributed under the terms of the Creative Commons Attribution License (http://creativecommons.org/licenses/by/2.0), which permits unrestricted use, distribution, and reproduction in any medium, provided the original work is properly cited.

\begin{abstract}
Background: The strikingly young age of new variant Creutzfeldt-Jacob disease (vCJD) cases remains unexplained. Age dependent susceptibility to infection has been put forward, but differential dietary exposure to contaminated food products in the UK population according to age and sex during the bovine spongiform encephalopathy (BSE) epidemic may provide a simpler explanation.
\end{abstract}

Methods: Using recently published estimates of dietary exposure in mathematical models of the epidemiology of the new variant Creutzfeldt Jacob disease ( $\mathrm{vCJD}$ ), we examine whether the age characteristics of $\mathrm{VCJD}$ cases may be reproduced.

Results: The susceptibility/exposure risk function has likely peaked in adolescents and was followed by a sharp decrease with age, evocative of the profile of exposure to bovine material consumption according to age. However, assuming that the risk of contamination was proportional to exposure, with no age dependent susceptibility, the model failed to reproduce the observed age characteristics of the VCJD cases: The predicted cumulated proportion of cases over 40 years was $48 \%$, in strong disagreement with the observed $10 \%$. Incorporating age dependent susceptibility led to a cumulated proportion of cases over 40 years old of $12 \%$.

Conclusions: This analysis provides evidence that differential dietary exposure alone fails to explain the pattern of age in vCJD cases. Decreasing age related susceptibility is required to reproduce the characteristics of the age distribution of $\mathrm{VCJD}$ cases.

\section{Background}

Owing to the lack of data concerning age dependent dietary exposure to bovine material in food in the UK, mathematical models used in the study of new variant Creutzfeldt-Jacob disease (vCJD) have postulated an age risk function for contamination where the influence of age dependent dietary exposure to contaminated material could not be separated from intrinsic age related suscepti- bility [1-3]. For example, we modelled exposure/susceptibility by a plateau during the first 15 years of age followed by an exponential decrease afterwards [2]. Using this approach we estimated the duration of the incubation period for vCJD to circa 15 years; and predicted that the epidemic had likely peaked in 2001-2002 which is now consistent with the observed data $[2,3]$. The simple representation of the age risk function implied that few 
parameters were necessary to describe it, a desirable property when estimation is based on limited data.

By June 2003, 139 cases of vCJD had been reported to the vCJD surveillance unit in the UK, as compared to the 79 cases used in our original paper [2]. This warrants the inclusion of more detail in the age risk function, especially to investigate whether the constant risk assumption in children holds, as it led to predict a bimodal age distribution for cases in the coming years [3]. At the same time, detailed estimates of the dietary bovine material consumption in the UK population according to age and sex have been made available [4]. This offers the opportunity to try to disentangle the role of exposure from that of agespecific susceptibility. Indeed, the estimates for dietary exposure show that it peaked during adolescence and decreased with age afterwards, a pattern which is consistent with the finding that most vCJD cases are young and were therefore at most teenagers during the years when the bovine spongiform encephalopathy (BSE) epidemic was at its maximum.

We therefore set out to estimate the age risk function with a versatile description based on step functions, and to investigate whether dietary exposure alone could explain the age distribution of cases.

\section{Methods}

\section{Data}

We obtained the age, sex and date of onset for the 137 vCJD cases reported to the UK vCJD unit as of June 2003, all of which had onset before October 2002. Delays in reporting may reach 18 months ( $\mathrm{R}$ Will, personal communication) and bias downward the incidence curve. Therefore, we included in our analysis only the 129 cases with onset before November 2001, consisting in 71 men and 58 women. Mortality by age and sex for the UK was obtained from the Office for National Statistics [5].

\section{Model}

The model extends our previously described method [3]. The instantaneous risk of infection for vCJD, $\lambda(a, t)$, was assumed to depend on date $t$ and age $a$ in a multiplicative manner, with $\lambda(a, t)=f(a) g(t)$, where $g(t)=\left\{\begin{array}{cc}\exp (0.596(t-1989)) & 1980<t<1989 \\ r \exp (-0.596(t-1989)) & 1989<t<1996\end{array}\right.$ parallels the entry of infected cows in the human food chain estimated from back-calculation results in the UK [6] and $r$ corresponds to the impact of the specified risk material ban in 1989; $f(a)$ is a function varying with age only (see following paragraph). Occurrence of cases of vCJD of sex $i$ ( $\mathrm{i}=$ Male or Female) is then modelled by a Poisson process in the (age, time) plane, with intensity $\pi_{i}(a, t)=\beta_{i} S_{i}(a, t) \int_{0}^{a}\left\{\exp \left[-\int_{0}^{v} \lambda_{i}(u, t-a+u) d u\right] \lambda_{i}(v, t-a+v) h(a-v) d v\right\}$ , so that the expected number of onsets of sex $i$ in the period $[t, t+\mathrm{d} t)$ and age $[a, a+\mathrm{d} a)$ is $\pi_{i}(a, t) \mathrm{d} a \mathrm{~d} t[2,7]$. In the equation above, $h$ is the distribution of the incubation time, $S_{i}(a, t)$ is the probability of survival at time $t$ for individuals of sex $i$ aged $a$ calculated from census information, and $\beta_{1}$ is the intensity of a homogeneous Poisson process. The incubation period distribution $h$ was taken to be lognormal, as the choice of a particular shape is not critical for the estimates [3]. This distribution was also assumed to be independent of sex, and of age at contamination.

Estimates of the parameters were obtained by numerical maximization of the log-likelihood, defined as $\sum_{i=1}^{n} \log \left[\pi_{s_{i}}\left(a_{i}, t_{i}\right)\right]-\sum_{s} \iint_{D} \pi_{s}(a, t) d a d t$, where the first sum is on all observed cases where $s_{i}$ is the sex of individual $i$, and the second is over both sexes $(s)$ and requires integration of the intensity over the domain (in time and age) $D=[0,100] \times[1$ Jan 1980,31 Nov 2001]. Custom FORTRAN code, using SLATEC and TOMS library for numerical routines, was used in this step. In all instances, our model allowed estimation of the mean and standard deviation of the incubation period, the shape of $f(a)$, and $\beta$.

The model predicted distribution of age for cases with onset before November 2001 was determined by integrat-

ing $\hat{\pi}(a, t)$ over the period [1/1/1980, 1/11/2001] for consecutive age class of width 10 years. The $\chi^{2}$ distance was computed to measure agreement between the observed and the model calculated age distributions, but no formal test was carried out.

\section{Age Risk function \\ Model "Susceptibility \& Diet"}

To allow a versatile shape in the age risk function, we chose a non parametric description based on step functions rather than a mathematical function with few parameters.

We wrote

$f(a)=\left\{\begin{array}{cc}f_{i}, & 5(i-1) \leq a<5 i, i=1, \cdots, 5 \\ f_{6} \exp (-\alpha(a-25)), & a \geq 25\end{array}\right.$ so that the shape of $f(a)$ was unconstrained for $a<25$ and decreased exponentially after age 25 . We then estimated $f_{i^{\prime}}$ $i=1, \ldots, 6$ and $\alpha$ at maximum likelihood. With this particular choice, it is possible to visually check the progression in risk with age during infancy $\left(f_{1}, f_{2}, f_{3}\right)$, the presence of a peak in risk in teenagers $\left(f_{2}, f_{3}, f_{4}\right)$, and whether the drop in risk after 20 is strong enough to be exponential $\left(f_{4^{\prime}} f_{5^{\prime}} f_{6}\right)$. 


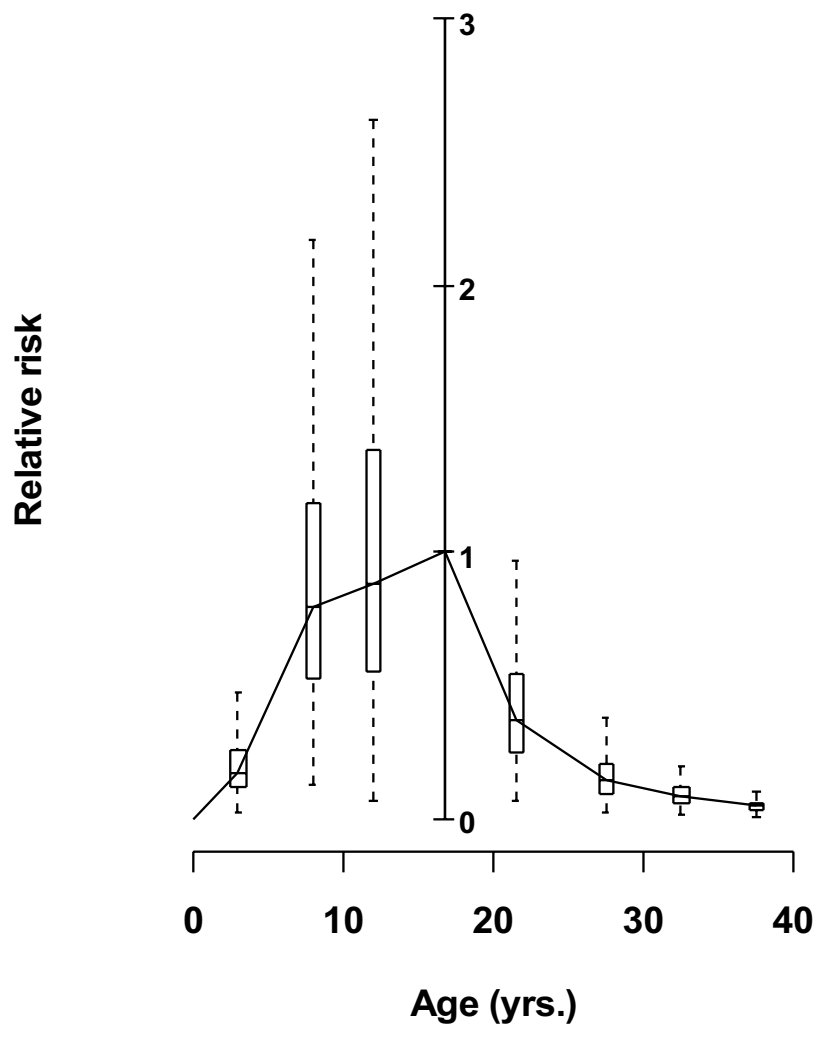

Figure I

Age dependent exposure/susceptibility risk function for vCJD infection. Risks are relative to the $[15,20]$ years old. Box and whiskers plots show the $50 \%$ and $95 \%$ confidence intervals.

\section{Model "Diet Alone"}

To investigate whether dietary exposure to meat was sufficient to explain the young age of vCJD cases, we first determined the exposure to meat according to age and sex from UK data, using consumption of carcass meat [4]. In these data, it is seen that the quantity consumed by week, as well as the percentage of consumers, changes with age. We therefore defined $e(a)$ as the product of mean consumption by the corresponding percentage of consumers in age class $a$, and wrote $f(a)=f_{0} e(a)$, where $f_{0}$ was estimated at maximum likelihood.

Model "Susceptibility | Diet"

Finally, to estimate the influence of age standardised on dietary exposure, we wrote $\left\{\begin{array}{cc}f_{i} e(a), & 5(i-1) \leq a<5 i, i=1, \cdots, 5 \\ f_{6} \exp (-\alpha(a-25)) e(a), & a \geq 25\end{array}\right.$ and likewise estimated $f_{i}$ from the data.
In model "Susceptibility \& Diet" and "Susceptibility | Diet", we normalised the $f_{i}$ by the largest of the values in the graphical presentation of results, yielding risks relative to the highest risk age class.

\section{Confidence intervals}

Maximum likelihood estimators have a limiting normal distribution around the true parameters, with limiting variance/covariance matrix given by the inverse of the Fisher information [8], therefore the quadratic form $Q(\theta)=(\theta-\hat{\theta})^{\prime} i(\theta)(\theta-\hat{\theta})$, where $\theta$ stands for a vector of $k$ parameters, $\hat{\theta}$ for the estimates, and $i(\theta)$ for the Fisher information matrix, has a limiting chi-squared distribution with $k$ degrees of freedom. We therefore determined 95\% confidence intervals by finding the values of parameters so that $Q(\theta)$ was less than the $95^{\text {th }}$ quantile of the corresponding chi-squared distribution.

\section{Results}

The estimated age risk function in model "Susceptibility \& Diet" is presented in Figure 1, and shows that an increase during childhood, peak during adolescence and sharp decrease afterwards provided the best fit. In this model, the average incubation period was estimated at 13.2 years (CI95\% [11.2, 15.8]), with standard deviation 2.0 years (CI95\% [1.1,3.7]). The model predicted age for the cases showed good agreement with the observed distribution $\left(\chi^{2}=2.45\right.$; Figure 2$)$.

The shape of the estimated age risk function in Figure 1 is evocative of the age profile of dietary exposure to bovine carcass meat in the 1980s in the UK, as shown in Figure 3, where an increase in consumption was noted during childhood and adolescence, and decreased afterwards. However, in model "Diet Alone", while the average incubation period was estimated at 12.1 (CI 95\% [10.2, 14.2]) years with a standard deviation of 2.4 (CI95\% [1.2, $4.1]) y e a r s$, close to that of the first model, the predicted age distribution of the cases was at odds with the observed distribution $\left(\chi^{2}=58.4\right)$, as apparent in Figure 2. More precisely, the predicted percentage of cases aged over 40 was $48 \%$ with the "Diet Alone" assumption, when the observed percentage was only $10 \%$; it was $12 \%$ with the "Susceptibility \& Diet" model.

When estimating the residual influence of age, once exposure has been taken into account, model "Susceptibility | Diet" led to a profile for the fi as shown in Figure 4. This profile retained the major characteristics of the age risk function obtained in the "Susceptibility \& Diet" model, although with an earlier maximum susceptibility. With this model, the average incubation period established at 12.6 years (CI 95\% [10.5, 14.7]) with standard error 1.8 


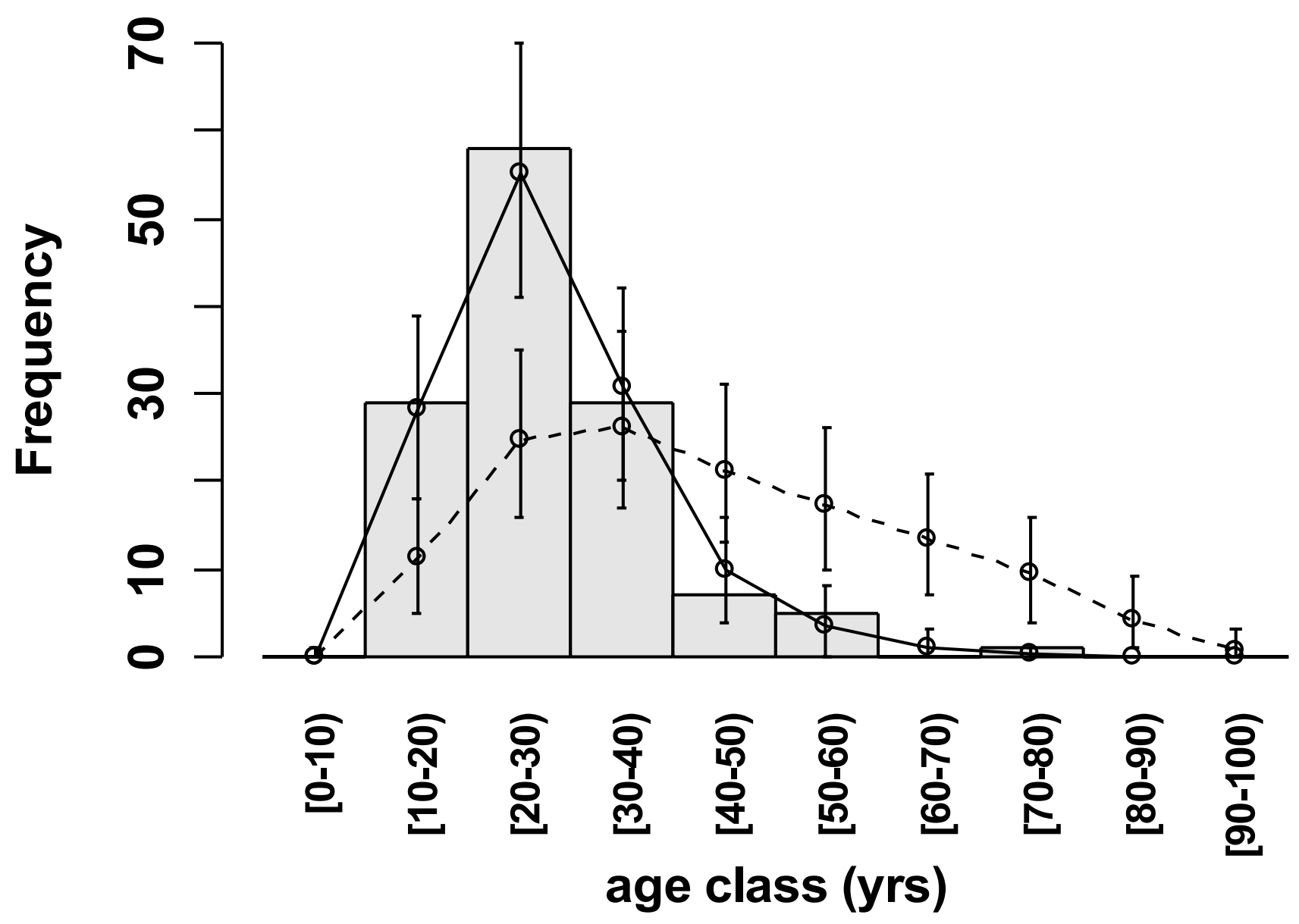

Figure 2

Observed age distribution of the I 29 vCJD cases with onset before November $200 \mathrm{I}$ and model predicted cumulated age distribution of cases for susceptibility proportional to meat consumption only (dashed) or with estimated age risk function (plain). Bars limit $95 \%$ confidence intervals.

years (CI95\% $[1.2,3.5])$, and the predicted age distribution of cases showed agreement similar to that of the "Susceptibility \& Diet" model $\left(\chi^{2}=2.36\right)$.

\section{Discussion}

Incorporating differential dietary exposure to BSE infected products according to age and sex, in a flexible age risk function for vCJD contamination, we found that exposure alone could not explain the young age of vCJD cases seen in the UK. Decreasing age related susceptibility had to be assumed to reproduce the characteristics of the age distribution of these cases.

In all instances, the estimates for the epidemiological characteristics of vCJD were in line with those previously reported from comparable number of cases $[3,9,10]$, and pointed to an epidemic of moderate size. We obtained a smaller point estimate for the mean incubation period when the age risk was allowed to change during childhood rather than assumed constant (13.2 yrs CI95\% $[11.2,15.8]$ vs. 16.4 yrs CI95\% [11, 24] [3]). All models considered here predicted that, by 2010, the epidemic should have ended, provided it is limited to individuals with the observed susceptible genotype (as of today, all vCJD cases are methionine homozygous at codon 129 of the PrP gene[11]). The average number of cases to come is predicted at 43 , leading to a total size of 172 cases.

From the model "Susceptibility \& Diet", it appears that a previous assumption of a constant age risk in children and adolescents [2] has likely led to overestimate the risk of infection in young children. The age-risk profile estimated 


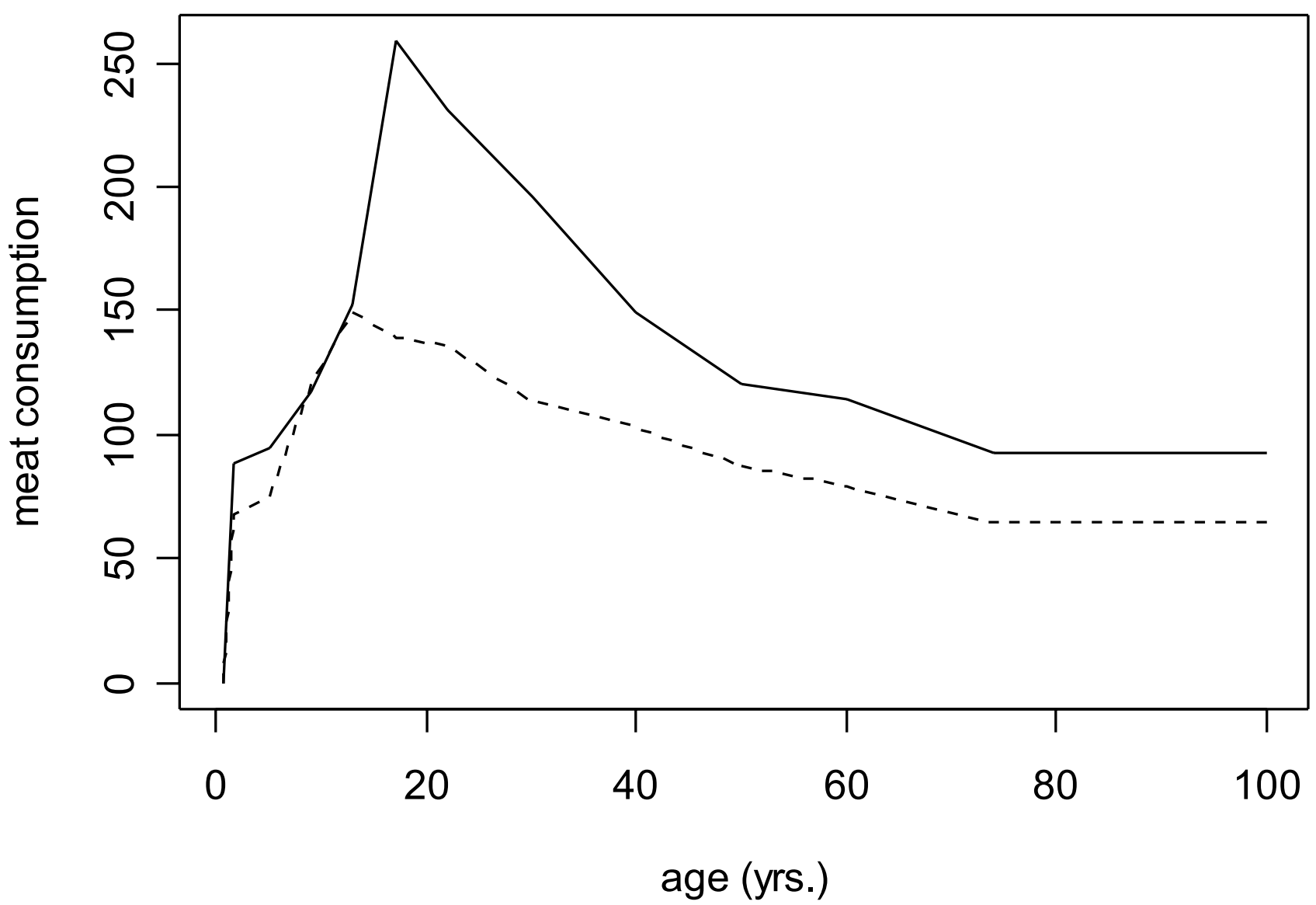

Figure 3

Average dietary exposure per person per week in the UK according to sex (plain : Male, dashed : Female). Data from Cooper \& Bird [4].

here leads to a smaller risk than previously found in children born after 1980, to fewer cases among the young and an overall smaller total size for the epidemic than reported before. Furthermore, since few young cases are expected in the future, the bimodality of the predicted age distribution of cases is not found anymore. The estimated profile of the susceptibility/exposure age risk function agreed with that selected in scenario analysis [9], although our maximal risk is among the 15-20 years old rather than in the $10-15$ years old.

However, even after adjustment for dietary exposure, susceptibility remains rapidly decreasing in adults, as found in the "Susceptibility | Diet" model. This finding confirms that obtained in a recent analysis based on scenario analysis, where it was found that exponential decrease in susceptibility in the oldest cohorts was desirable [10]. This is also found here by direct estimation, and moreover, the age susceptibility appears to increase among children up to age 5-10, be almost constant among teenagers and rapidly decreasing afterwards.

Three hypotheses have been put forward to explain the young age of the vCJD cases: age dependent incubation period, age dependent exposure, and age dependent susceptibility. Age dependent incubation period has recently been revived by Cooper et al., because scenarios including longer incubation periods in old cohorts provided a better fit of the data[10]. However, the increase of the mean age of cases with time which should occur with age dependent incubation periods [2], is not supported by the data. The correlation between age at onset and calendar time remains indeed extremely small (Spearman correlation coefficient $\mathrm{r}=-0.025, n=129$ ). 


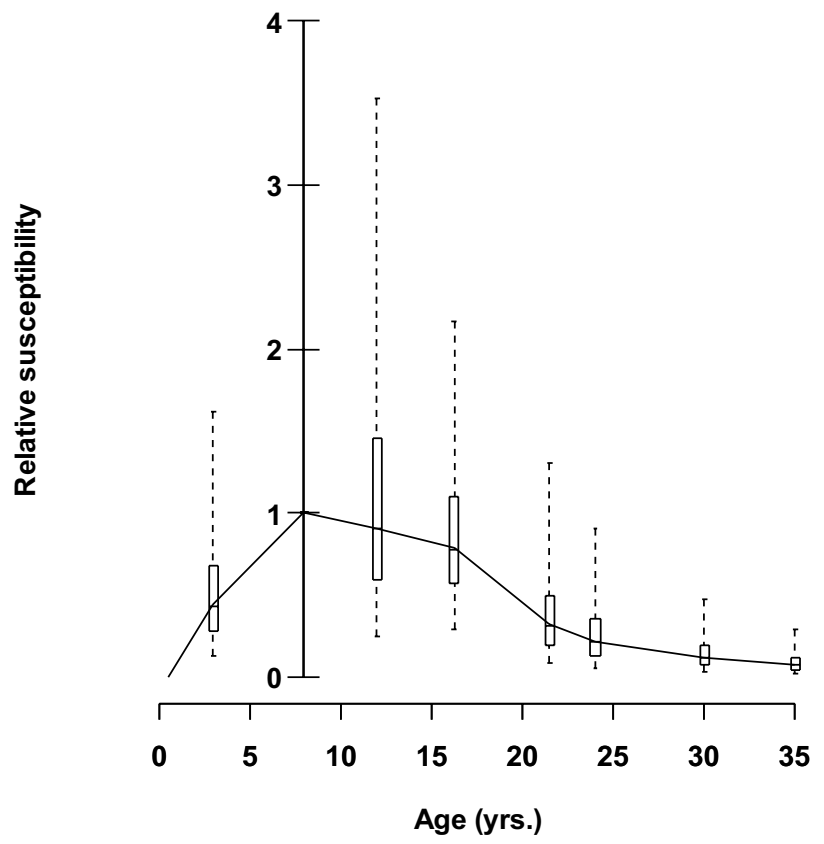

Figure 4

Age dependent susceptibility risk function for vCJD infection adjusted on dietary exposure to bovine material. Risks are relative to the $[5,10]$ years old. Box and whiskers plots show the $50 \%$ and $95 \%$ confidence intervals.

The potential role of differential dietary exposure was proposed early in the epidemic [12], because substantial differences in exposure existed in adolescents and adults. A disequilibrium in sex ratio towards males was for example predicted on this basis [13], but is not statistically established today ( $\chi^{2}$ test, $\mathrm{P}=0.49$ ) although the proportion of males is $55 \%$. Our analysis shows that, while the estimated age risk function for infection with vCJD exhibits a peak in the young that was also found in dietary exposure to potentially BSE infected products, differential dietary exposure alone does not account for the observed pattern in the age of the vCJD cases. Indeed, the relative exposure does not decrease rapidly enough with age to reduce the risk in older adults. Therefore, an additional effect of age is required to fully account for the age of the vCJD cases. Once exposure is taken into account, this effect appears to be peaking in children less than 10 years old, and decreasing afterwards.

Contamination through BSE infected food is today the most plausible explanation for the occurrence of vCJD; this is the rationale for correcting the risk of infection by the level of dietary exposure. However, this explanation remains today hypothetical, because even if epidemiology and biochemistry favour a link between BSE and vCJD, this is not regarded as ultimately conclusive [14]. In this work, we examined the further possibility that, provided dietary exposure was the culprit, differences in age-related exposure could explain the age distribution of cases. This assumes that the risk of vCJD infection may have been larger in those consuming more bovine products; however population based models linking bovine products consumption to vCJD incidence were not conclusive in this respect [15].

\section{Conclusions}

The reasons for an increased susceptibility in teenagers compared to young children and adults are today speculative. In many infectious diseases, a decrease in susceptibility with age is mediated through the immunological responses to repeated exposure since birth. However, for vCJD, most cohorts in the population have had the same duration of exposure to BSE infected material. Due to the age range considered, biological processes involved in the maturation of the immune system; in response to hormonal changes may be incriminated. For example, changes in the permeability of the intestinal barrier occurs with age with decreasing Peyer's patches [16]. Further experimental research is required to provide explanations for what remains a very unusual characteristic in an infectious disease.

\section{List of abbreviations}

vCJD: variant Creutzfeldt-Jacob Disease

BSE : Bovine Spongiform Encephalopathy

\section{Competing interests}

None declared.

\section{Authors' contributions}

PYB designed the study, analysed the results and drafted the manuscript. JYC participated in the design and writing. AJV participated in the design and writing. All authors read and approved the final manuscript.

\section{Acknowledgements}

Robert Will for allowing access to vCJD data.

Jean Gagnon for helpful discussions.

\section{References}

I. Ghani AC, Ferguson NM, Donnelly CA, Anderson RM: Predicted vCJD mortality in Great Britain. Nature 2000, 406:583-584.

2. Valleron AJ, Boelle PY, Will R, Cesbron JY: Estimation of epidemic size and incubation time based on age characteristics of vCJD in the United Kingdom. Science 200I, 294: 1726-I728.

3. Boelle PY, Thomas G, Valleron AJ, Cesbron JY, Will R: Modelling the epidemic of variant Creutzfeldt-Jakob disease in the UK based on age characteristics: updated, detailed analysis. Stat Methods Med Res 2003, I 2:221-233. 
4. Cooper JD, Bird SM: UK bovine carcass meat consumed as burgers, sausages and other meat products: by birth cohort and gender. J Cancer Epidemiol Prev 2002, 7:49-57.

5. Office for National Statistics: Tables 6.I - Deaths : age and sex. Population Trends 2004, I I 6:47.

6. Donnelly CA, Ferguson NM, Ghani AC, Anderson RM: Implications of BSE infection screening data for the scale of the British BSE epidemic and current European infection levels. Proc $R$ Soc Lond B Biol Sci 2002, 269:2179-2190.

7. Keiding N: Age-specific Incidence and Prevalence: a Statistical Perspective. J R Stat Soc Ser A Stat Soc 199I, I 54:37I-4I2.

8. Cox DR, Hinkley DV: Theoretical Statistics. Volume I. Ist edition. London, Chapman \& Hall; 1974:5 II.

9. Ghani AC, Donnelly CA, Ferguson NM, Anderson RM: Updated projections of future vCJD deaths in the UK. BMC Infect Dis 2003, 3:4.

10. Cooper JD, Bird SM: Predicting incidence of variant Creutzfeldt-Jacob disease from UK dietary exposure to bovine spongiform encephalopathy for the 1940 to 1969 and post | 969 birth cohorts. Int J Epidemiol 2003, 32:784-79I.

II. Will RG, Ward HJ: Clinical features of variant CreutzfeldtJakob disease. Curr Top Microbiol Immunol 2004, 284: I II-I32.

12. Gore SM, Bingham S, Day NE: Age related dietary exposure to meat products from British dietary surveys of teenagers and adults in the 1980s and 1990s. Br Med J 1997, 3 I 5:404-405.

13. Cooper JD, Gore SM: Should we anticipate male excess in vCJD cases? J Epidemiol Biostat 1999, 4:61-63.

14. Aguzzi $A$, Polymenidou M: Mammalian prion biology: one century of evolving concepts. Cell 2004, I | 6:3 | 3-327.

15. Cousens S, Everington D, Ward HJ, Huillard J, Will RG, Smith PG: The geographical distribution of variant Creutzfeldt-Jakob disease cases in the UK: what can we learn from it? Stat Methods Med Res 2003, I 2:235-246.

16. Van Kruiningen HJ, Ganley LM, Freda BJ: The role of Peyer's patches in the age-related incidence of Crohn's disease. J Clin Gastroenterol 1997, 25:470-475.

\section{Pre-publication history}

The pre-publication history for this paper can be accessed here:

http://www.biomedcentral.com/1471-2334/4/26/prepub

Publish with Biomed Central and every scientist can read your work free of charge

"BioMed Central will be the most significant development for disseminating the results of biomedical research in our lifetime. "

Sir Paul Nurse, Cancer Research UK

Your research papers will be:

- available free of charge to the entire biomedical community

- peer reviewed and published immediately upon acceptance

- cited in PubMed and archived on PubMed Central

- yours - you keep the copyright 